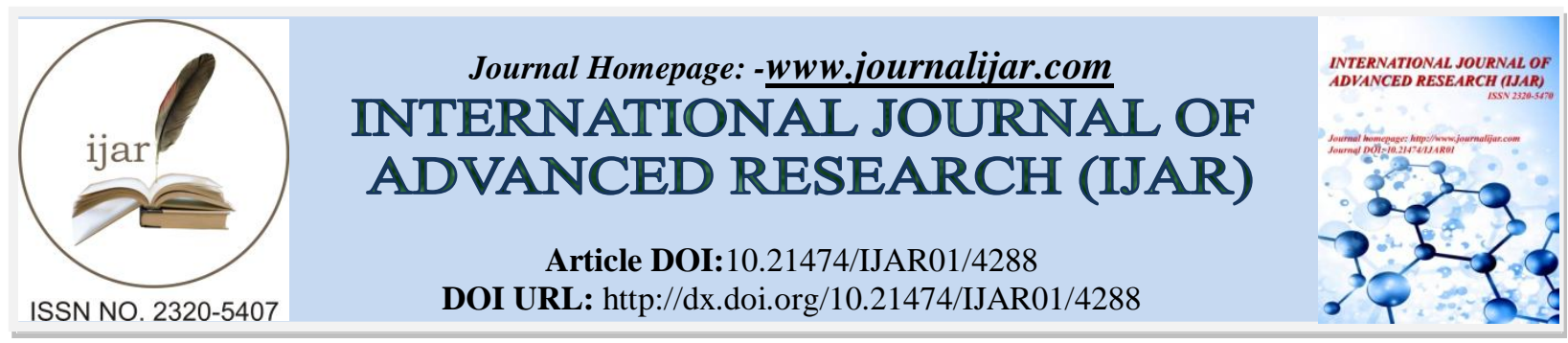

RESEARCH ARTICLE

\title{
PRACTICES FOLLOWED FOR COMPLETE DENTURE IMPRESSIONS AMONG GENERAL DENTAL PRACTITIONERS- A SURVEY.
}

Naisargi P. Shah, Pankti N. Shah, Sabita M. Ram and Jyoti B. Nadgere.

\section{Manuscript Info}

Manuscript History

Received: 28 March 2017

Final Accepted: 30 April 2017

Published: May 2017

Key words:-

Complete denture impressions, survey, trends in impression making

\section{Abstract}

Aim: To determine the practices followed for complete denture impressions among general dental practitioners.

Materials and Methods: The survey questionnaire consisted of 13 questions for dental practitioners to determine the practices followed for complete denture impressions. This questionnaire was validated by 10 dental practitioners and was sent to 600 general dental practitioners in Mumbai and Navi Mumbai fabricating complete dentures for patients.

Conclusion: Irreversible hydrocolloid is the most commonly used primary impression material. Majority of the practitioners advocate the use of selective pressure technique for final impression. The trend of using a custom tray with spacer and providing relief holes still prevails. Low fusing impression compound and zinc oxide eugenol paste are the most preferred final impression material amongst dental practitioners. Majority of the dentists establish the posterior palatal seal area at the border molding stage. However, more awareness needs to be created to follow proper disinfection protocols.

Clinical significance: Since there are numerous theories and philosophies of complete denture impression making, there is high variation in the method and materials followed by general dental practitioners. This survey will enable us to know the procedures followed by general dental practitioners during complete denture impressions and give us information about their belief and understanding of the influence of the method and materials used during complete denture impression making on the health of the oral tissues and on the retention, stability and support of the complete denture prosthesis.

Copy Right, IJAR, 2017,. All rights reserved.

\section{Introduction:-}

Complete denture is one of the most common prosthetic treatment option offered to a completely edentulous patient. Although the average age of onset of complete edentulism has been significantly delayed because of better dental healthcare facilities and increased awareness, a large number of edentulous patients seek dental rehabilitation to improve their quality of life. Though implant therapy has become a treatment option for edentulous patients, the long treatment duration, increased cost and possible systematic disorders may increase the need for conventional complete dentures ${ }^{1}$. Even if a completely edentulous patient is rehabilitated with implants, it's planning requires 
fabrication of a complete denture as the initial step. Complete denture remains the base on which the implant placement and prosthetic rehabilitation is done for the edentulous patient.

Before starting the process of complete denture fabrication, it is important to take a detailed case history and perform meticulous oral examination. If the patient is a previous denture wearer, the old dentures should be evaluated and certain precautions must be taken before fabrication of the new dentures is started.

Impression making is a very critical step in the fabrication of complete denture. It plays a major role in the denture's stability, support, retention, comfort and maintenance of good health of oral tissues.

The three basic schools of thought for conventional impression making in complete denture are mucostatic technique, mucocompressive technique and selective pressure technique. The amount of pressure applied on the underlying tissue while making the impression and the tissue displacement that occurs because of it differs between these three techniques. A variety of materials and methods are recommended in academics and are being followed at various stages of the complete denture fabrication like preliminary impression, custom tray fabrication, border molding, final impression and so on. Because of this variety, the general dental practitioner is faced with a varied choice of materials and techniques for impressions for complete dentures. This variety suggests that no single technique can be completely satisfactory for varied clinical situations. Different clinicians follow different techniques for the same problem. ${ }^{2}$

This survey will give us an idea of the practices followed for complete denture fabrication by general dental practitioners in Mumbai and Navi Mumbai.

\section{Materials and Methods:-}

A survey was conducted among the General Dental practitioners practicing in and around Mumbai and Navi Mumbai and fabricating complete dentures for patients. This was in context with which methods are being following for complete denture impressions. This survey was approved by the Research Committee and Institutional Ethics Committee. The survey questionnaire consisted of 13 questions regarding the practices followed for complete denture fabrication. After validating the questionnaire from 10 dental practitioners, it was sent to 600 general dental practitioners in Mumbai and Navi Mumbai fabricating complete dentures for patients. The forms were collected and the results were analyzed

\section{Survey Form:-}

Before starting with the complete denture fabrication procedure, do you take the patient's case history and perform oral examination?
a. Yes
b. No

2. How much time before making an impression do you ask the patient to discontinue the use of previous denture?
a. less than 48 hours
b. 48 hours-72hours
c. more than 72 hours
d. No discontinuation

3. Which material do you use for making a primary impression?
a. Impression compound
b. Irreversible hydrocolloid
c. Putty elastomeric impression material

4. Do you make a final impression for a complete denture?
a. Yes
b. No
if no, proceed to question 12

5. Do you provide a spacer while fabricating a custom tray?
a. Yes
b. No

6. Do you give tissue stop while fabricating the custom tray?
a. Yes
b. No

7. Which material do you use for fabrication of custom tray?
a. Shellac base plate
b. Self cure resin 

c. Light cure resin
d. Vacuum forming sheet

e. Old dentures.

8. Do you carry out border molding procedure?

a. Yes

b. No

9. Which material do you use to carry out border molding procedure prior to final impression?
a. Low fusing compound (green stick)
b. Self cure resin
c. Elastomeric impression material
d. Zinc oxide eugenol impression paste

10. Do you make relief holes in the custom tray before making the wash impression?
a. Yes
b. No

11. Which material do you use for making the final impression?
a. Zinc oxide eugenol impression paste
b. Irreversible hydrocolloid
c. Elastomeric impression material
d. Tissue conditioners

12 .At what stage do you establish the posterior palatal seal?
a. Primary impression stage
c. On the master cast
b. Border molding stage
d. Not specific

13. Which agent do you use for disinfecting the impression?
a. Glutaraldehyde
b. Iodophores
c. Chlorine compounds
d. Phenolics

e. No disinfection

\section{Results:-}

Out of the 600 survey forms sent 546 were received back accounting to a response rate of $91 \%$. The answers were analysed and the percentage of the answers were calculated. According to this survey, before starting with the complete denture fabrication procedure, all the dentists take the patient's case history and perform oral examination (Graph 1). 58.44\% dentists ask the patient to discontinue the use of previous denture 48-72 hours before making an impression, $22.07 \%$ dentists ask the patient to discontinue its use for less than 48 hours, $11.68 \%$ of the dentists do not ask the patients to discontinue its use while $7.79 \%$ of the dentists ask the patient to discontinue its use for more than 72 hours before making an impression (Graph 1). A majority of 79.22\% of the dentist use irreversible hydrocolloid for making the primary impression while $12.98 \%$ use impression compound and $7.79 \%$ of the dentists use putty elastomeric impression material for making primary impression (Graph 1). According to this survey, all dentists make a final impression for a complete denture (Graph 2). Amongst them, a spacer is provided by $93.5 \%$ while fabricating a custom tray while no spacer is provided by $6.49 \%$ (Graph 2). Also tissue stops while fabricating the custom trays are provided by $62.33 \%$ whereas no tissue stops are provided by $37.66 \%$ (Graph 2). Fabrication of custom tray with self cure resin is done by $73.5 \%$ of the dentists, with light cure resin by $12.98 \%$ and with shellac baseplate by $11.68 \%$ (Graph 2). Border molding procedure is carried out by all dentists (Graph 3) out of which low fusing impression compound (green stick) is used by $89.61 \%$ dentists, zinc oxide eugenol paste is used by $5.19 \%$, elastomeric impression material is used by $3.89 \%$ and self cure resin is used by $1.29 \%$ (Graph 3). Relief holes in the custom tray before making the wash impression is made by $98.70 \%$ dentists while no relief holes are made by $1.29 \%$ (Graph 3). Final impression is made using zinc oxide eugenol paste by $52.38 \%$ dentists, with irreversible hydrocolloid by $33.3 \%$ and with elastomeric impression material by $14.28 \%$ (Graph 4). The posterior palatal seal area is established at the border molding stage by maximum dentists $(77.9 \%)$, at primary impression stage by $18.18 \%$ dentists, on the master cast by $1.29 \%$ dentists while $2.59 \%$ do not establish it at any specific stage (Graph 4). Disinfection of the impression is done using glutaraldehyde by $36.36 \%$ dentist, chlorine compounds by $2.59 \%$ dentists, iodophores by $1.29 \%$ dentists while no disinfection of impression is done by $59.74 \%$ dentists (Graph 4). 
Graphs:-

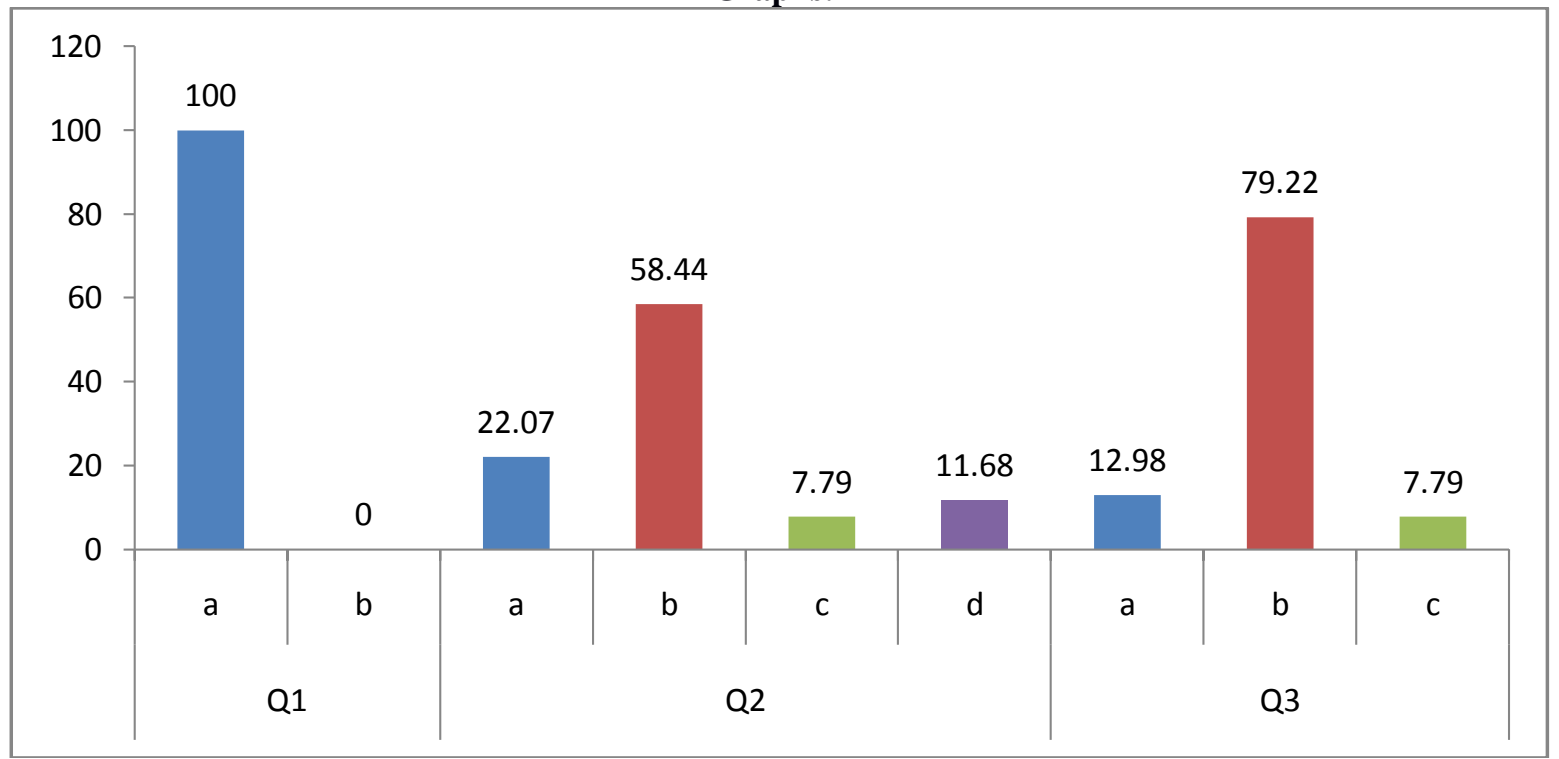

Graph1

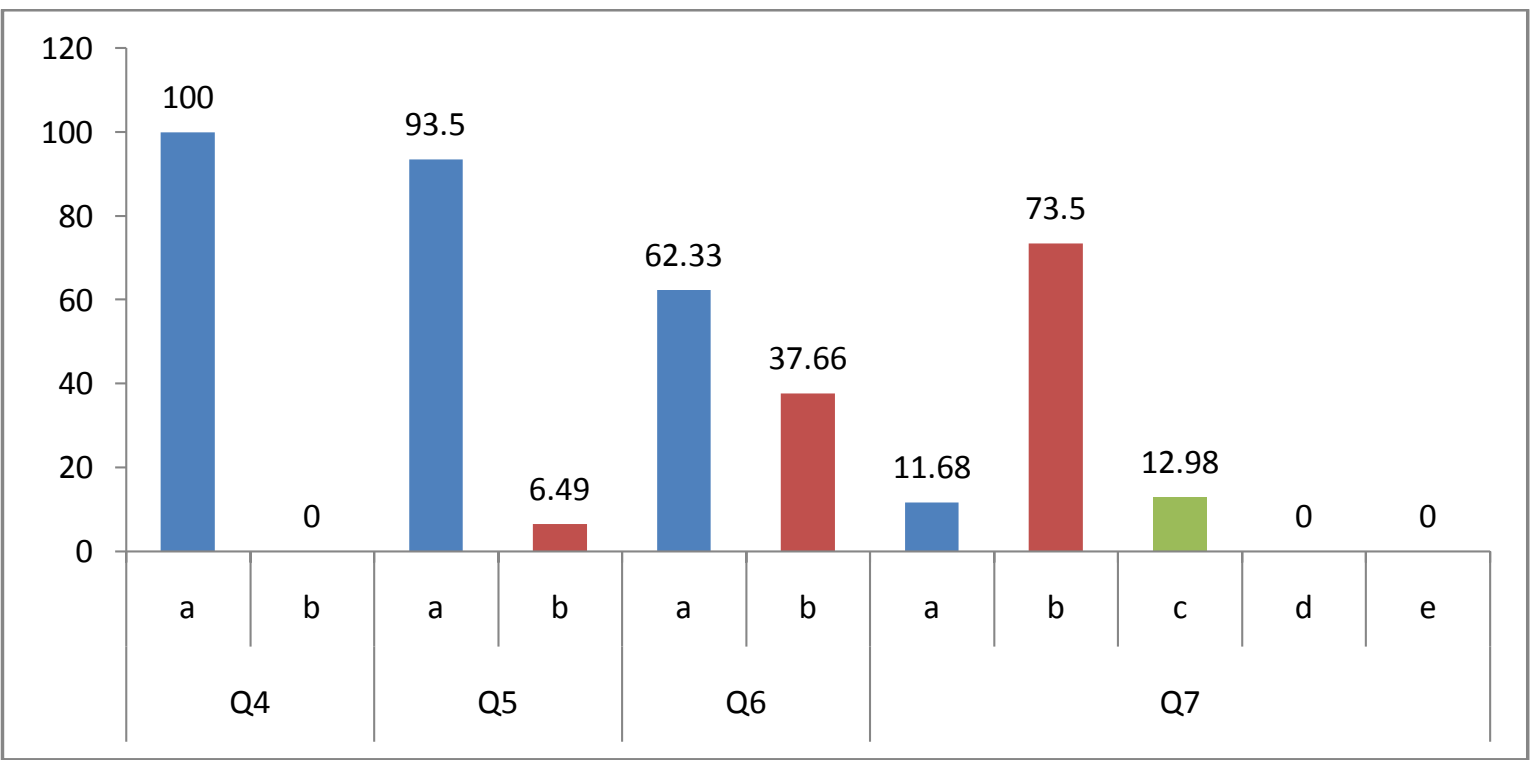

Graph 2 


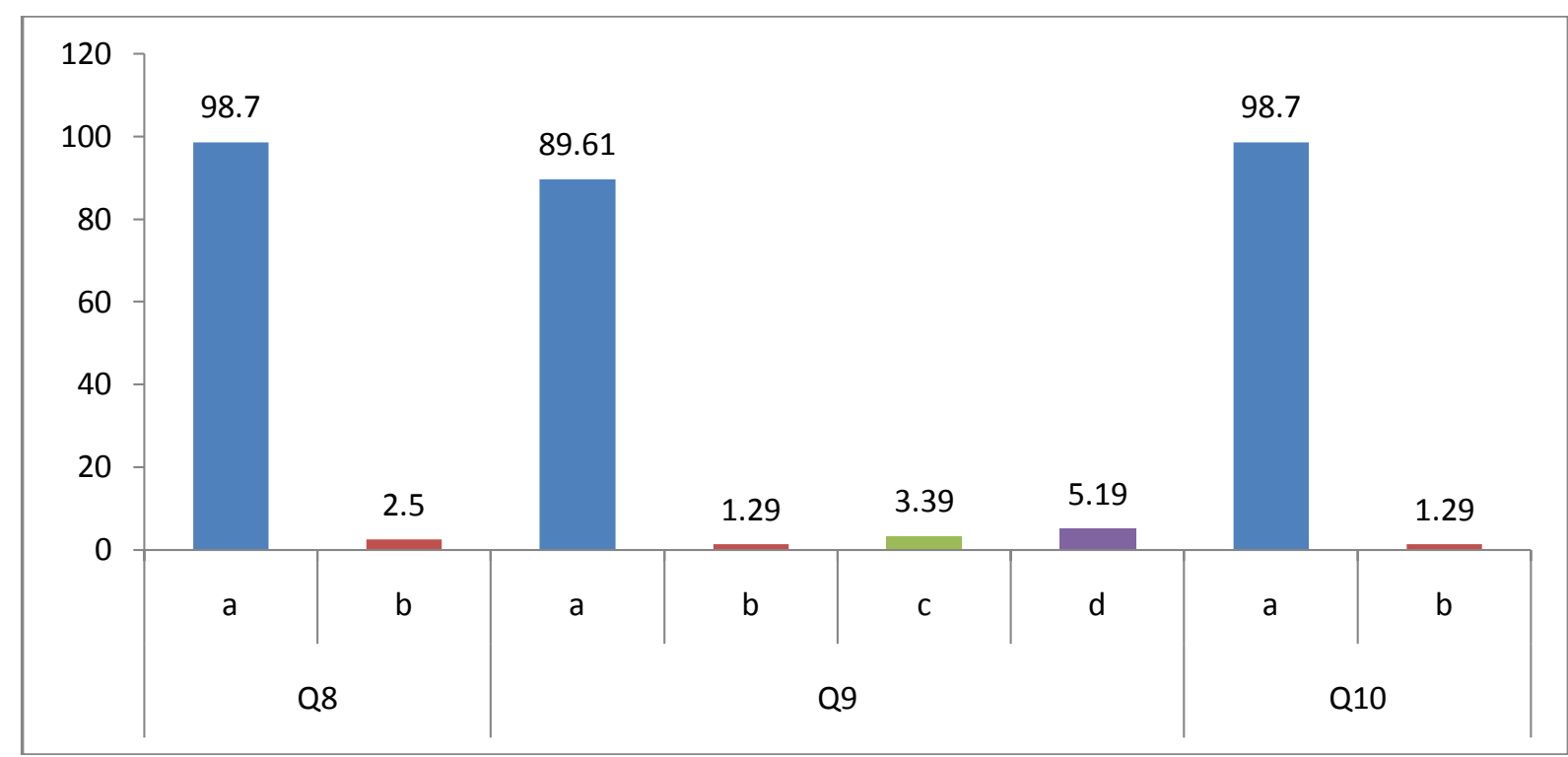

Graph 3

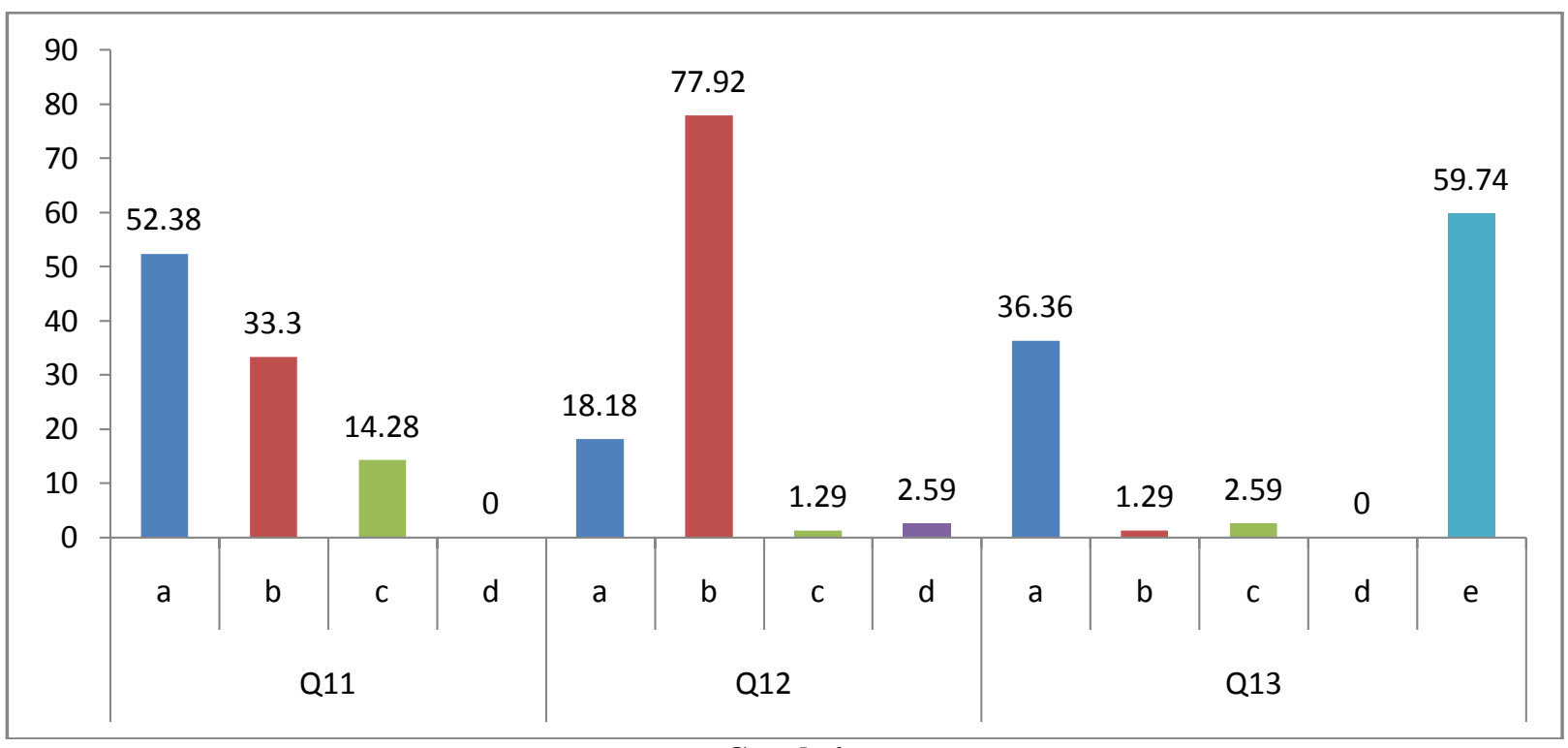

Graph 4

\section{Discussion:-}

This survey was conducted to recognize the current trends being followed for complete denture impressions among general dental practitioners of Mumbai and Navi Mumbai.

Taking a detailed case history helps establish a positive professional relationship with the patient. When paired with a thorough oral examination, it provides the clinician with information of patient's past medical, dental \& personal history. It also gives the clinician an idea about the patient's expectations, mental attitude and financial condition. All these help the clinician in making proper diagnosis and treatment planning best suited for the patient. According to this survey all the dentists take case history and perform oral examination prior to starting with the treatment procedure. This is an extremely essential step and should not be omitted.

Distortion of the supporting tissues occurs if the patient has been wearing ill fitting complete dentures. This was demonstrated by Lytle in $1957^{3}$. Before fabrication of the new dentures it is essential for the tissues of the basal seat to be healthy and of suitable contour. This is most effectively achieved by asking the patient to stop wearing the old 
dentures. In this survey maximum clinicians $(88.32 \%)$ ask the patient to discontinue use of their previous denture with $58.44 \%$ asking the patient to discontinue the use $48-72$ hours prior to impression making while a substantial $11.68 \%$ dentists do not ask the patient to discontinue its use at all.

Making a good primary impression lays a foundation for a good final impression. This depends largely on the clinicians' skill and the impression material used. The most commonly used preliminary impression material in this survey was irreversible hydrocolloid (79.22\%), while only $12.98 \%$ used impression compound. This is consistent with a survey carried out in Indian private practitioners ${ }^{4}$ and with a survey by Singh $\mathrm{G}$ et al in four major Indian cities showed that a majority (71\%) use alginate for preliminary impressions ${ }^{5}$. Also a survey in US dental schools and in United Kingdom show a majority of use of irreversible hydrocolloid as the primary impression material of choice $^{6-8}$.

Final impression records the shape of the supporting mucosa over the alveolar ridges and hard palate along with functional depth and width of sulci as accurately as possible. This leads to better retention, stability and support of the complete denture. In this survey all the dentists make a final impression for the fabrication of complete denture.

Out of these a high majority of $93.50 \%$ provide a spacer before the fabrication of custom tray. There are mainly three basic schools of thought for impression making namely the mucostatic technique, the mucocompressive technique and the selective pressure technique. By providing a spacer, relief is provided to the non stress bearing area while the stress bearing areas are recorded under slight placement of pressure.

Acccording to literature tissue stops help in proper vertical seating of the impression tray \& controls thickness of impression material ${ }^{9}$. In this survey $62.3 \%$ provide tissue stops in the custom tray.

Visible light cure resin have advantages like accuracy of fit, superior strength, complete polymerization without residual compounds, absence of free methyl methacrylate, color stability, ease of fabrication, and ease of manipulation ${ }^{10}$. However in this only $12.98 \%$ dentists use light cure resin to fabricate custom tray. Majority of the dentist (75.32\%) use acrylic resin while $11.68 \%$ still continue to use shellac base plate for fabrication of custom tray. Base plate is not recommended since it will be highly unstable during the procedure as it is a thermoplastic material.

Recording the sulcus area in terms of depth and width and the tissue surface is important in order to ensure adequate retention. This is obtained by proper border molding followed by a final impression. In this survey $98.70 \%$ practitioners perform border molding procedures.

Low fusing impression compound as a material of border molding has several advantages like dimensional stability, ability to place it incrementally, ability to readapt it by heating and low cost. In this survey $89.61 \%$ dentist continue to use low fusing impression compound (green stick) for border molding On the other hand elastomers have the advantage of being less time consuming as the border molding is done in a single stage and errors in one section will not affect the subsequent sections. In this survey $5.19 \%$ use zinc oxide eugenol paste for border molding. However $3.89 \%$ use elastomeic impression material and $1.29 \%$ use self cure resin for border molding. This is consistent with a survey in Gujarat in which $88 \%$ used low fusing impression compound for border molding and while $4 \%$ used elastomeric impression materials including polyvinylsiloxane and polyether ${ }^{11}$. Use of elastomeric materials for border molding was reported slightly high $(17 \%)$ in a survey conducted in four Indian cities ${ }^{4}$.

Relief holes help the excess impression material to escape and also help relieve the hydrostatic pressure created during the final impression procedure and prevent voids in the impression. In this survey about $98.70 \%$ practitioners make relief holes in the custom tray before making the wash impression and $1.29 \%$ do not make relief holes

In this survey, $52.38 \%$ use zinc oxide eugenol paste to make the final impression while $14.28 \%$ use elastomeric impression material while $33.3 \%$ dentist used irreversible hydrocolloid for final impression. This is consistent with a survey conducted in Gujarat ${ }^{11}$. However zinc oxide eugenol is capable of recording the minor details of the tissue, is mucostatic, economical and dimensionally stable. But it has certain disadvantages like it is inelastic and thus cannot be used in situations where there are deep undercuts, it may cause a burning sensation and sticks to the patients skin. On the other hand elastomers have the advantages like ease of handling, dimensional stability, elastic recovery. However it has the disadvantage of being expensive. 
The posterior palatal seal area is one of the most critical areas responsible for the retention of the maxillary denture. Establishing and recording this area is extremely crucial and should not be ignored. Maximum dentists (77.9\%) establish the posterior palatal seal at the border molding stage, and $18.18 \%$ at primary impression stage. Only $1.29 \%$ dentists establish it on the master cast. This method is arbitrary and should not be advocated.

It is necessary to disinfect the impression before sending the impression or the cast it to the laboratory. In this survey a substantial $59.74 \%$ of the dentists do not disinfect the impressions at all. This may cause to be a health hazard to the dental assistants, the lab technician and the dentist. Out of the remaining $40.26 \%$ dentist who disinfect the impressions, $36.36 \%$ dentists use $2 \%$ glutaraldehyde for the same while $2.59 \%$ use chlorine compounds and $1.29 \%$ use iodophores

With the introduction of intraoral scanners, the need of impression for fabrication of the prosthesis may be eliminated but this technology is expensive and more research needs to be done to evaluate its superiority to the conventional impression making technique.

\section{Conclusion:-}

Within the limitations of this survey, the following conclusions can be drawn after the analysis of the results:

1. Irreversible hydrocolloid is the most commonly used primary impression material.

2. Majority of the practitioners advocate the use of selective pressure technique for final impression.

3. The trend of using a custom tray with spacer and providing relief holes still prevails.

4. Low fusing impression compound and zinc oxide eugenol paste are the most preferred final impression material amongst dental practitioners.

5. Majority of the dentists establish the posterior palatal seal area at the border molding stage. However it should be established and recorded at the primary impression stage itself.

6. More awareness needs to be created to follow proper disinfection protocols.

\section{Summary:-}

Complete denture is one of the most common removable treatment option for a completely edentulous patient. Even for rehabilitation using fixed prosthesis, complete denture fabrication plays an important role in the planning for the same. Impressions are a critical step and influence the retention, stability and support of the denture. Several concepts and material are available for impression making. Different clinicians advocate use of different methods for the same clinical situation. Also, there is not enough awareness about proper impression disinfection protocols amongst the dentist.

\section{References:-}

1. Zitzmann NU, Sendi P, Marinello CP. An economic evaluation of implant treatment in edentulous patients-preliminary results. Int J Prosthodont 2005; 18: 20-27.

2. Hyde TP, McCord JF. Survey of prosthodontic impression procedures for complete dentures in general dental practice in the United Kingdom. J Prosthet Dent. 1999 Mar;81(3):295-9.

3. Lawson A. Current concepts and practice in complete dentures impressions: principles and practice.J of Dentistry 1978; 6(1): 43-58.

4. Kakatkar VR, Complete denture impression techniques practiced by private dental practitioners: a survey, J Indian Prosthodont Soc, 13(3), 2013, 233-235.

5. Singh G, Kapoor V, Gambhir R and Bansal V, Application of prosthodontic techniques by private practitioners in Northern India- a survey, Internet Journal of Epidemiology, 9(2), 2010.

6. Hyde TP and McCord JF, Survey of prosthodontic impression procedures for complete dentures in general dental practice in the United Kingdom, J Prosthet Dent, 81(3), 1999, 295-299.

7. Petropoulos VC and Rashedi B, Complete denture education in U.S.dental schools, J Prosthodont, 14(3), 2005, $191-197$.

8. Mehra M, Vahidi F and Berg RW, A complete denture impression technique survey of postdoctoral prosthodontic programs in the United States, J Prosthodont, 23(4), 2014, 320-327.

9. P. W. Smith, R. Richmond, J. F. McCord. The design and use of special trays in prosthodontics: guidelines to improve clinical effectiveness . British dental journal: 1999 October 23; 187(8).

10. Ogle RE, Sorensen SE and Lewis EA, A new visible light-cured resin system applied to removable prosthodontics. J Prosthet Dent, 56(4), 1986, 497-506.

11. Shah R, Lagdive S, Barajod P, Patel M. Complete Denture Impression Procedures and Techniques Practiced by Dentists across the State of Gujarat: A Survey. IOSR J of Dent and Medical Sciences2015 Jun; 14(6): 01-11. 